\title{
The Biological-Behavioral Effect Of Neuritin On Non-Small Cell Lung Cancer Vascular Endothelial Cells Via VEGFR And Notch I
}

This article was published in the following Dove Press journal: OncoTargets and Therapy

\author{
Qiao Zhang' \\ Juan Zhang ${ }^{2}$ \\ Jian Zhang ${ }^{3}$ \\ Patiguli Aerxiding' \\ Amina Quhai' \\ Cuncun Chen ${ }^{4}$ \\ Li Shan' \\ 'Department of Thoraciconcology, The \\ Third Affiliated Hospital of Xinjiang \\ Medical University, Tumor Hospital \\ Affiliated to Xinjiang Medical University, \\ Urumqi, Xinjiang 8300II, People's \\ Republic of China; ${ }^{2}$ Department of \\ Respiratory and Critical Care Medicine, \\ First People's Hospital of Kashgar \\ Xinjiang 844000, People's Republic of \\ China; ${ }^{3}$ Health Corps of the People's \\ Liberation Army 69260 Troops, Urumqi, \\ Xinjiang, 830002, People's Republic of \\ China; ${ }^{4}$ Department of Thoracic Surgery, \\ Chest Hospital of Xinjiang Uyghur \\ Autonomous Region, Urumqi, Xinjiang \\ 830049, People's Republic of China
}

Correspondence: Cuncun Chen Department of Thoracic Surgery, Chest Hospital of Xinjiang Uyghur Autonomous Region, Urumqi, Xinjiang 830049, People's Republic of China

Tel +099l-7500419

Fax +86099I-7860085

Email chenpuyingxiong@yeah.net

Li Shan

Department of Thoraciconcology, The

Third Affiliated Hospital of Xinjiang

Medical University, Tumor Hospital

Affiliated to Xinjiang Medical University,

Urumqi, Xinjiang 8300II, People's

Republic of China

Tel $+86 / 3609989394$

$\mathrm{Fax}+860991-7819342$

Email shanlinew319@I63.com
Purpose: This study aims to elucidate the biological behavior of Neuritin abnormal expression in pulmonary vascular endothelial cells (VECs) of non-small cell lung cancer (NSCLC), and explore its possible underlying mechanisms.

Patients and methods: Primary NSCLC-VECs were isolated from 10 cancer tissues from NSCLC patients, purified and identified by CD34 and Factor VIII staining. Real-time PCR and Western-blot were adopted for detecting the expression levels of Neuritin, Notch1, and VEGFR in NSCLC-VECs and HPMECs. Neuritin-overexpression, Neuritin-knockdown NSCLC-VECs and HPMECs were constructed by transfection of pcDNA3, 1-Neuritin vector, and pBS/U6-Neuritin siRNA. Changes in cell proliferation, migration, cell cycle, and apoptosis were determined by using the MTT assay, scratch assay, transwell migration assay, and flow cytometry, respectively. Post-transfection changes in cell morphology were examined by scanning electron microscopy.

Results: The expression of Neuritin in NSCLC-VECs was significantly higher compared to that in HPMECs $(\mathrm{p}<0.01)$. Overexpression of Neuritin increased the expression of VEGFR while it reduced the expression of Notch1 $(\mathrm{p}<0.01)$; it also promoted cell proliferation, scratch healing, and in vitro migration $(\mathrm{p}<0.05)$ in HPMECs and NSCLC-VECs cells. Additionally, overexpression of Neuritin stimulated cell cycle progression and inhibited apoptosis in HPMECs and NSCLC-VECs $(\mathrm{p}<0.001)$. Under electron microscope, the pseudopodium of cell surface was obvious, indicating that the intercellular adhesion was upregulated. However, knockdown of Neuritin in HPMECs and NSCLC-VECs played exactly the opposite roles.

Conclusion: Neuritin was key in the progression of NSCLC through its biological activities, including anti-apoptosis, promoting VEC proliferation, migration, and cell cycle progression. Neuritin may affect its biological activity by positively regulating VEGFR expression and negatively regulating Notch1 signaling. Neuritin may serve as a potential biomarker for NSCLC.

Keywords: neuritin, non-small cell lung cancer, Notch1, VEGF

\section{Introduction}

Lung cancer was reported to be one of the most malignant cancers and the leading cause of cancer-related deaths with the highest morbidity and mortality in the world $^{1}$. While non-small cell lung cancer (NSCLC) is the main subtype of lung cancer, which accounts for $80-85 \%$ of the total lung cancer and its incidence has elevated in recent years. ${ }^{2,3}$ Furthermore, NSCLC is featured with poor prognosis and low 5-year survival. A majority of NSCLC patients are in the middle or 
advanced stage and over $50 \%$ of the patients present with metastatic disease at the time of diagnosis. ${ }^{4}$

The study of related molecular markers, including VEGF and Notch1, provides new therapeutic targets for NSCLC. ${ }^{5}$ Angiogenesis was demonstrated to be crucial in tumor growth and metastasis which has been widely studied in the treatment of various cancers. ${ }^{6-8}$ Anti-angiogenic therapy has provided novel insights and methods for targeted therapy of multiple tumors. Vascular endothelial growth factor (VEGF) and its receptors (VEGFR) are proangiogenic factors which play an important role in pathological angiogenesis and are closely related to the occurrence, development, invasion as well as metastasis of malignant tumors. ${ }^{9,10}$ In addition, abnormal expression of Notch signal pathway has already been confirmed to be connected with various solid tumors including NSCLC. However, their underlying mechanism remains unclear. ${ }^{11,12}$

Neuritin, as a neurotrophic factor associated with neuroplasticity, is highly expressed in many human tumors. ${ }^{13}$ It has been shown that Neuritin acted as a downstream factor for neurotrophins in the nervous system. ${ }^{14}$ Besides, it could promote neuronal migration and neuronal regeneration, inhibit neuronal apoptosis and consolidate the formation of synaptic circuits. ${ }^{15}$ According to cancer-related research, it contributes to stimulating human umbilical vein endothelial cells by recombining and accelerating endothelial cell migration as well as angiogenesis in tumor tissue. ${ }^{16}$ In addition, Neuritin can be used as a molecular marker for tumor hypoxia in multiple cancers consisting of muscle tumors and liver cancer. ${ }^{17}$ It has also been demonstrated that Neuritin inhibited Notch signaling. ${ }^{18}$ Nevertheless, its role and mechanism of NSCLC has not been reported.

The present study investigated whether Neuritin could regulate VEGFR and Notch 1 expression and affect its biologic activities in human NSCLC-vascular endothelial cells (NSCLC-VECs).

\section{Materials And Methods Clinical Data Of Patients}

Patients who were diagnosed with NSCLC and underwent surgery at the Department of Lung and Mediastinal Surgery of the Affiliated Tumor Hospital of Xinjiang Medical University between September and December 2017 were enrolled in this study. Lung cancer tissues were collected during surgeries. All patients signed the informed consent form, and the study was approved and supervised by the ethics committee of Xinjiang Medical University.

\section{Isolation, Purification, And Identification Of NSCLC-VECs}

Five to ten fresh lung cancer tissues were repeatedly washed with PBS to remove the blood and necrotic tissue. Then, the lung tissues were cut into $0.5 \mathrm{~mm} \times 0.5 \mathrm{~mm} \times 0.5 \mathrm{~mm}$ cubes, homogenized using a glass homogenizer and filtered through sterile 200 mesh filters. Residual tissues on the filter were transferred into flasks, digested with $0.2 \%$ trypsin at room temperature for $90 \mathrm{~min}$, then to be filtered by sterile $200 \mathrm{mesh}$ filters. Capillary membrane tissues were chosen and placed in F12 culture medium (JKChem, Shanghai, China) supplemented with $100 \mathrm{U} / \mathrm{mL}$ heparin (EGTA, Beijing, China) and $10 \%$ fetal bovine serum (FBS) (Hyclone, Rockford, IL, USA) and incubated at $37^{\circ} \mathrm{C}$ with $5 \% \mathrm{CO}_{2}$. Culture medium was replenished frequently until endothelial cells were sprouted from the tissue. NSCLC-VECs that grew from the tissues were observed and purified under an inverted microscope. Later, NSCLC-VECs from the second and fourth passage were identified by CD34 and Factor VIII IHC test kit (Yansheng, Shanghai, China) following the manufacturer's instructions. PBS was used as a negative control.

\section{Cell Culture}

The purified NSCLC-VECs and human pulmonary microvascular endothelial cells (HPMECs, EK-Bioscience, Shanghai, China) were cultured in 1640 medium containing 10\% FBS (Hyclone, Rockford, IL, USA) in a humidified atmosphere of $5 \% \mathrm{CO}_{2}$ at $37^{\circ} \mathrm{C}$. A total of $2-4$ generation cells were taken for subsequent experiments.

\section{Real-Time PCR}

Total RNA was extracted from NSCLC-VECs and HPMECs by using TRIzol reagent (Invitrogen, Carlsbad, CA, USA) as manufacturer's protocol stipulates. Reverse transcription kit (TaKaRa, Dalian, China) was applied for cDNA reversion. The mRNA levels of GAPDH, Neuritin, VEGF, and Notch1 were measured by quantitative PCR (qPCR) kit (TaKaRa, Dalian, China) according to the manufacturer's instructions. PCR conditions were as follows: initial denaturation at $95^{\circ} \mathrm{C}$ for 5 mins, 40 cycles of $95^{\circ} \mathrm{C}$ for $1 \mathrm{~min}$ (denaturing), $55^{\circ} \mathrm{C}$ for 2 mins (annealing), and $72^{\circ} \mathrm{C}$ for $1 \mathrm{~min}$ (extension), followed by $95^{\circ} \mathrm{C}$ for $15 \mathrm{~s}, 60^{\circ}$ $\mathrm{C}$ for $1 \mathrm{~min}$, and $95^{\circ} \mathrm{C}$ for $15 \mathrm{~s}$ for melting curve analysis. Primers are shown in Table 1. Each sample was tested in 4 
Table I Primer Sequences

\begin{tabular}{|c|c|}
\hline $\begin{array}{l}\text { Name Of } \\
\text { Primer }\end{array}$ & Primer Sequence \\
\hline GAPDH & $\begin{array}{l}\text { Forward:5'-CATTGGCTACGAATACAGCA-3' } \\
\text { Reverse:5'-AGGGGCAACTGGTCTACATG-3' }\end{array}$ \\
\hline Neuritin & $\begin{array}{l}\text { Forward:5'-ACAGCCCTTACGGATTGCCA-3' } \\
\text { Reverse:5'-GCTGCCGCAGAGTTCGAATAA-3' }\end{array}$ \\
\hline VEGF & $\begin{array}{l}\text { Forward:5'-GCCGCCTCTGTGGAGAATGA-3' } \\
\text { Reverse:5'-ACCСТTTGCTCACTGCCACT-3' }\end{array}$ \\
\hline Notch I & $\begin{array}{l}\text { Forward:5'-TTCTGCCTGACCTGGACGAC-3' } \\
\text { Reverse:5'-ATCCAGGTGCTGCTGAGTCC-3' }\end{array}$ \\
\hline
\end{tabular}

replicate wells, and experiments were performed in triplicate. A negative control was included in each experiment to determine the presence or absence of PCR contamination and dimers. The PCR reactions were performed in triplicate and the data were analyzed by $2-\Delta \Delta \mathrm{Ct}$.

\section{Cell Transfection And Morphology Observation}

The eukaryotic pcDNA3.1-Neuritin overexpression plasmid, pBS/U6-Neuritin siRNA interference plasmid, and negative control (NC) plasmid were constructed. Cells were seeded into 6-well plates one day before transfection. When cells reached $70-80 \%$ confluency, culture medium was removed and cells were washed once with PBS, followed by addition of Opti-MEM culture medium. Transfection was performed in line with the instructions of the Lipofectamine ${ }^{\mathrm{TM}} 2000$ transfection reagent (Invitrogen, Carlsbad, CA, USA). After $24-48 \mathrm{hrs}$ of culturing at $37^{\circ} \mathrm{C}$, culture medium was replaced with fresh complete culture medium containing $2 \mathrm{~g} / \mathrm{mL}$ puromycin, and cells were maintained for 3-5 passages until stable transfected cell lines were obtained. Changes in the morphology of transfected HPMECs and NSCLC-VECs were observed by scanning electron microscope (Oxford, INCA).

\section{MTT Assay}

Cells were seeded into 96-well plates at a density of $5 \times 10^{3}$ cells/well. MTT ( $5 \mathrm{mg} / \mathrm{mL}, 100 \mu \mathrm{L}$; Aladdin, China) was added to each well at $24 \mathrm{hrs}, 48 \mathrm{hrs}, 72 \mathrm{hrs}, 96 \mathrm{hrs}$, or 120 hrs post-seeding, and incubated for $4 \mathrm{hrs}$ at $37^{\circ} \mathrm{C}$ as Jue $\mathrm{Xu}$ et al described. ${ }^{19}$ Then, DMSO was added for 30 mins to dissolve MTT crystals. Optical density (OD) was measured using a 96-well microplate reader (DNM-9606, Perlong Medical) at $570 \mathrm{~nm}$. For each group, the mean OD value of 5 replicate wells was calculated.

\section{Flow Cytometry Analysis}

Cells were fixed overnight at $4^{\circ} \mathrm{C}$ in $70 \%$ ice-cold ethanol. For cell cycle analysis, cells were stained with PI from Cell cycle kits (Beyotime, China) based on the manufacturer's instructions. Cells were analyzed on FACScan Analytic Flow Cytometer (BD Bioscience, San Jose, California, USA). Cell cycle distribution was analyzed by using the Modfit software (Bio-Rad, Hercules, CA, USA).

For cell apoptosis analysis, cells were fixed and then stained with Annexin V-FITC from Annexin-V-FITC cell apoptosis kit (Beyotime, China) and then stained with PI. Cells were analyzed on a flow cytometer (BD Bioscience). Cell apoptotic rate was calculated by the ratio of early and late apoptotic cells to total cells.

\section{Scratch Assay}

Cells were seeded into 6-well plates. When cells reached $90 \%$ confluency, a vertical line was scratched down the middle of the well using a $10 \mu \mathrm{L}$ pipette tip. Culture medium was removed. Cells were washed $2-3$ times in PBS and then cultured in low serum or serum-free culture medium. At $48 \mathrm{hrs}$ post-scratch, wound healing was examined and imaged in each well.

\section{Transwell Migration Assay}

Cells were seeded into transwell inserts (Corning, NY, USA) at $2 \times 10^{4}$ cells/insert. Subsequently, serum-free culture medium was added into each insert to a final volume of $200 \mu \mathrm{L}$. Transwell inserts were placed into the wells (upper chamber) of a 24-well plate, and $600 \mu \mathrm{L}$ of complete culture medium was supplemented into the upper chamber. Each group was tested in triplicate. After incubation for $36 \mathrm{hrs}$, culture medium was detached from each insert/well, cells were washed 3 times in PBS and fixed in ice cold $75 \%$ ethanol for 30 mins. Upon removal of ethanol, inserts were air-dried, stained with crystal violet for 5-10 mins. Excess stain was washed later. Cells that migrated across the pores to the bottom side of the filter were visualized under the microscope. Cells in 5 random fields of view per well were counted. The assay was repeated 3 times to calculate the mean number of migrated cells in each group.

\section{Western Blot Analysis}

Cells were lysed in RIPA lysis buffer on ice for $30 \mathrm{mins}$, centrifuged at $12,000 \mathrm{r} / \mathrm{min}$ for $30 \mathrm{~min}$ at $4^{\circ} \mathrm{C}$, and the supernatant was collected. The amount of protein was 
determined using BCA protein assay kit (Thermo Fisher, San Jose, CA, USA). Proteins were separated by $12 \%$ SDS-PAGE and transferred onto a PVDF membrane. Primary antibodies, including anti-Notch1 (sc-376403), anti-VEGFR (sc-7269), anti- $\beta$-actin (sc-58673), and antiNeuritin (sc-365538) were purchased from Santa Cruz. The membrane was washed with TBST lasting 5 mins for three times, then to be incubated with the secondary goat anti-Rabbit IgG (1:1000 dilution; Protech, Asia) marked with HRP for $1 \mathrm{hr}$ under $37^{\circ} \mathrm{C}$ and washed. The bands were visualized with an enhanced chemiluminescence (ECL) system (Tanon, Shanghai, China), and protein levels were quantified using Image-Pro Plus 6.0 (Media Cybernetics, Inc., Rockville, MD, USA).

\section{Statistical Analysis}

The experimental results were analyzed by SPSS 19.0 software (IBM Corp., Armonk, NY, USA). Variance analysis was used after normality analysis and homogeneity test of variance followed by the Tukey's Honest Significant Difference post hoc test. All results were presented as mean \pm SD. All functional assays were performed in triplicate. $\mathrm{p}<0.05$ was considered statistically significant.

\section{Results}

\section{Primary NSCLC-VECs Identification}

To identify the isolated cells, two specific markers of endothelial cells, CD34 and Factor VIII were stained. Merging images showed that both CD34 and factor VIII were expressed in the isolated primary NSCLC-VECs (Figure 1), suggesting that primary cells are purified successfully, thus can be used for subsequent experiments.

\section{Neuritin Is Highly Expressed In NSCLC-VEC Cells}

The expression of Neuritin protein and mRNA in NSCLCVECs and HPMECs was evaluated by Western blot analysis and qPCR. In accordance with the result, the Neuritin expression in NSCLC-VECs was notably higher than that in normal HPMECs $(\mathrm{p}<0.01)$ (Figure 2A and B), revealing that Neuritin level was upregulated in pulmonary vascular endothelial cells of NSCLC patients.

\section{Neuritin Promotes VEGFR And Inhibits Notch I Expression}

To determine the effect of Neuritin on VEGFR and Notch1 expression, Western blot analysis and qPCR were
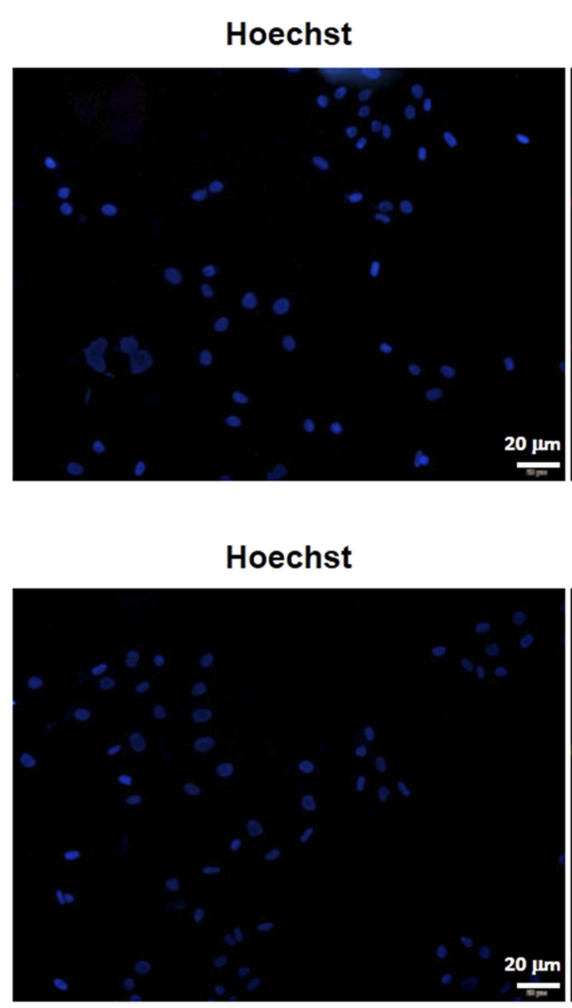

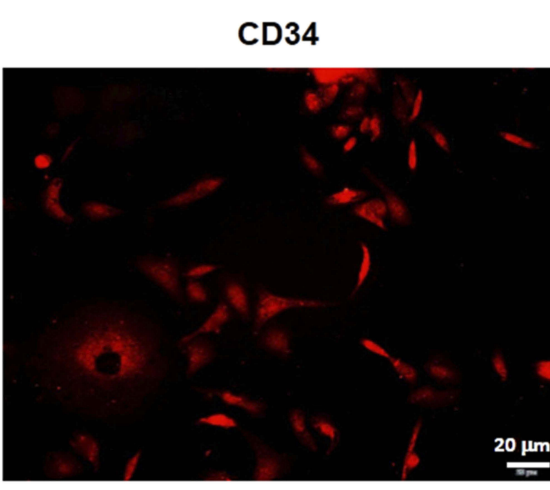

Factor VIII

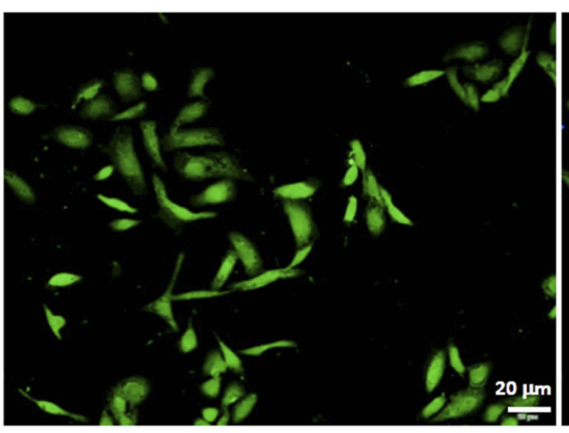

Merge

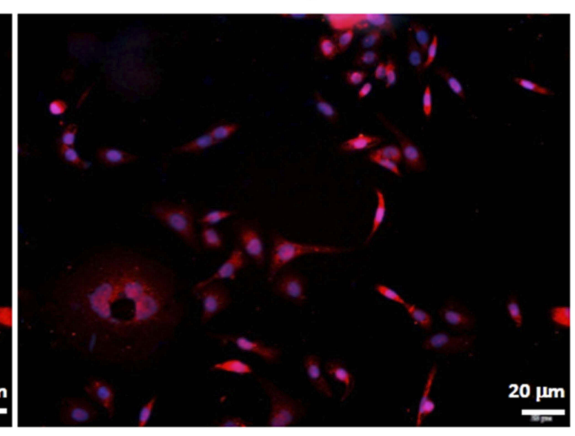

Merge

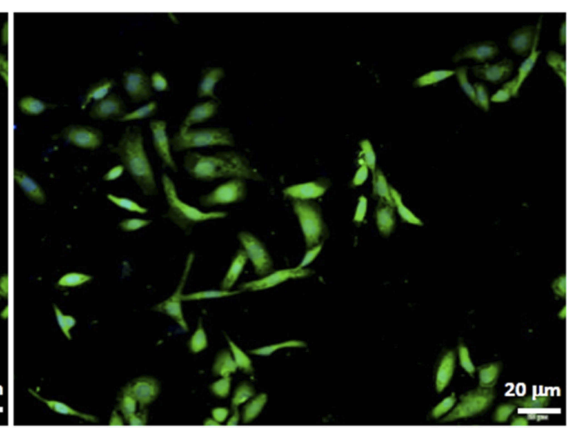

Figure I Identification of NSCLC-VECs. Cells were stained by Hoechst, CD34, and Factor VIII and detected by confocal laser scanning microscopy. Scale bar $20 \mu \mathrm{m}$. 
A
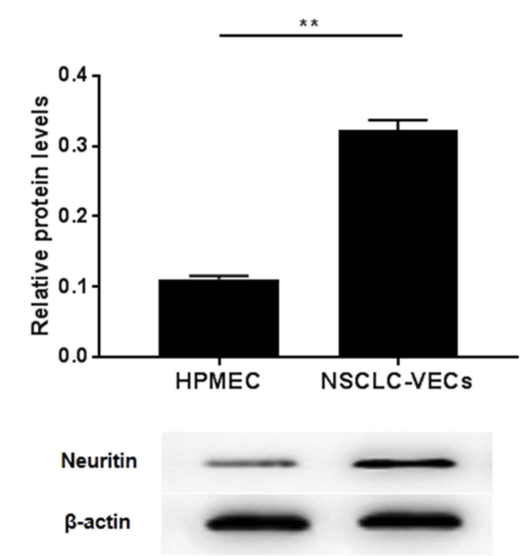

B

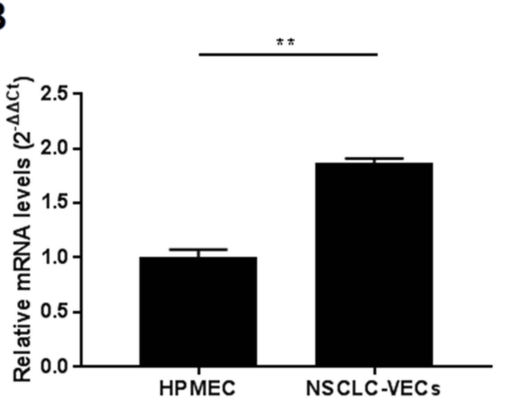

C
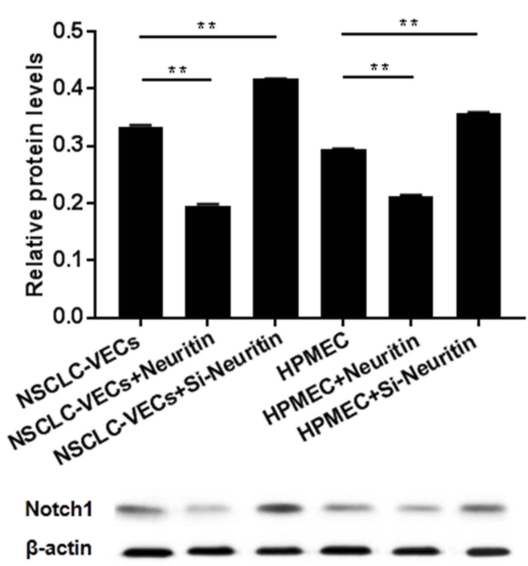

D

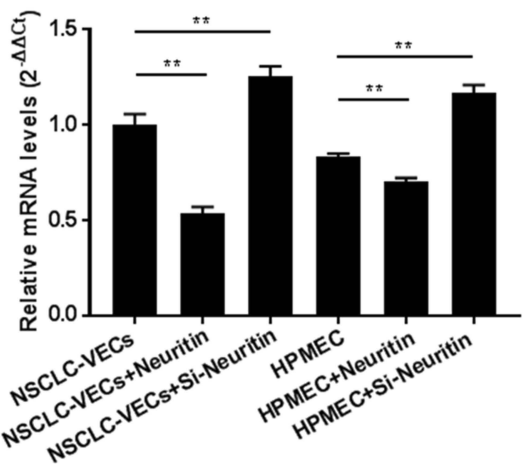

E
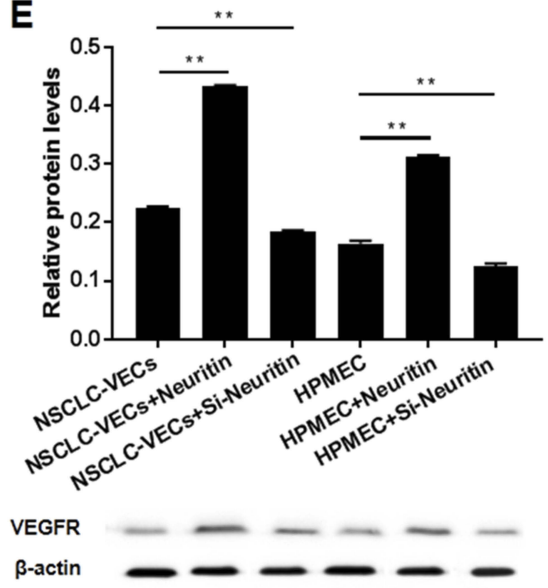

F

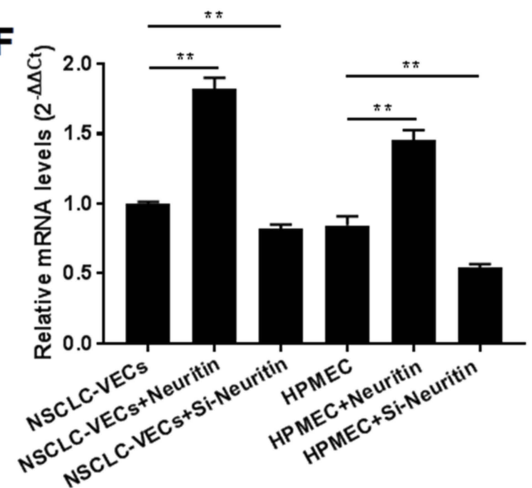

Figure 2 Expression level of Neuritin, VEGFR, and NotchI in HPMEC and NSCLC-VECs cells. (A) The expression level of Neuritin protein in HPMEC and NSCLC-VECs cells was detected by Western blot. (B) The relative expression level of Neuritin mRNA in HPMEC and NSCLC-VECs cells was detected by qRT-PCR. Compared with HPMEC, ${ }^{* *}$ p $<0.01$. (C-F) Neuritin was overexpressed or silenced in HPMECs and NSCLC-VECs. Expression of Notch I protein (C), NotchI mRNA (E), VEGFR protein (D), and VEGFR mRNA (F) was detected. Compared with NC group, **p<0.0I.

performed after Neuritin overexpression or knockdown in HPMECs and NSCLC-VECs. Our findings showed that the mRNA and protein of Notch1 were obviously descended $(\mathrm{p}<0.01)$ in both HPMECs and NSCLC-VECs by Neuritin overexpression but markedly ascended when Neuritin was knocked down $(\mathrm{p}<0.01)$ (Figure 2C and D). Conversely, VEGFR was a vascular regulatory factor whose expression was opposite to Notch1 $(\mathrm{p}<0.01)$ (Figure 2E and F). These results indicated that Neuritin negatively regulated Notch1 signaling pathway and had a positive regulatory effect on VEGFR expression in VECs.

\section{Neuritin Promotes Proliferation And Migration Of VECs}

The effect of Neuritin on cellular behavior of HPMECs and NSCLC-VECs was determined after Neuritin overexpression or knockdown. From the results, at $72 \mathrm{hrs}$ after post-adhesion, cell proliferation (Figure 3A and B), wound healing (Figure 3C), and cell migration (Figure 3D) were particularly enhanced in Neuritin overexpressing cells in contrast with $\mathrm{NC}$ (all $\mathrm{p}<0.05)$. Whereas, cell proliferation, wound healing, and migration were significantly suppressed in Neuritin knockdown cells when compared to the NC group (both $\mathrm{p}<0.05$ ). Consequently, Neuritin promoted the proliferation and migration of VECs.

\section{Neuritin Affects Cell Cycle Distribution And Inhibits Apoptosis Of VECs}

The cell cycle distribution and apoptosis of HPMEC and NSCLC-VECs were detected by flow cytometry after inhibition or overexpression of Neuritin. Knockdown of Neuritin promoted G0/G1 cell cycle arrest while reduced $\mathrm{S}$ phase cell distribution (Figure $4 \mathrm{~A}-\mathrm{C}$ ). On the contrary, Neuritin overexpression significantly accelerated cell cycle progression into the $\mathrm{G} 2$ phase and $\mathrm{S}$ phase (Figure 4A-C). In addition, apoptosis of HPMECs and NSCLC-VECs had an obvious rise by Neuritin interference but significantly reduced by Neuritin overexpression 
A

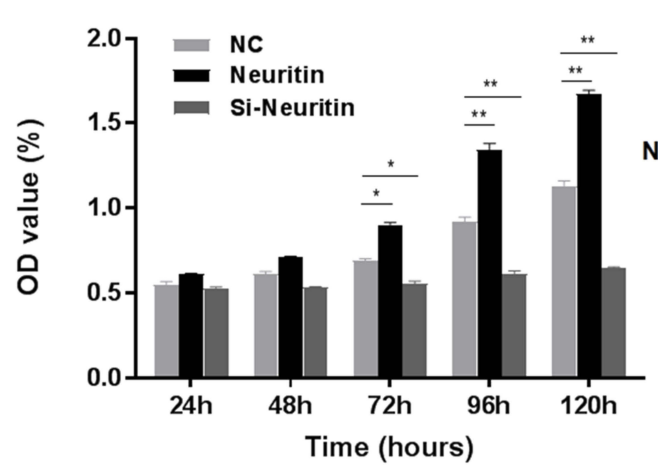

C

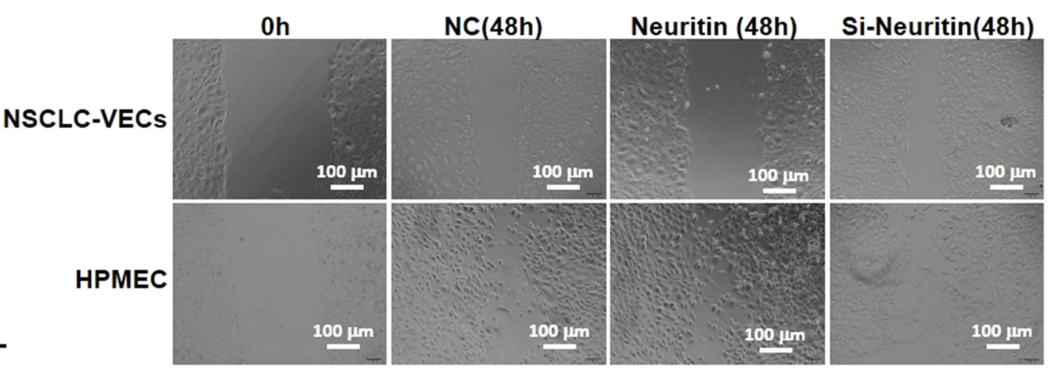

D

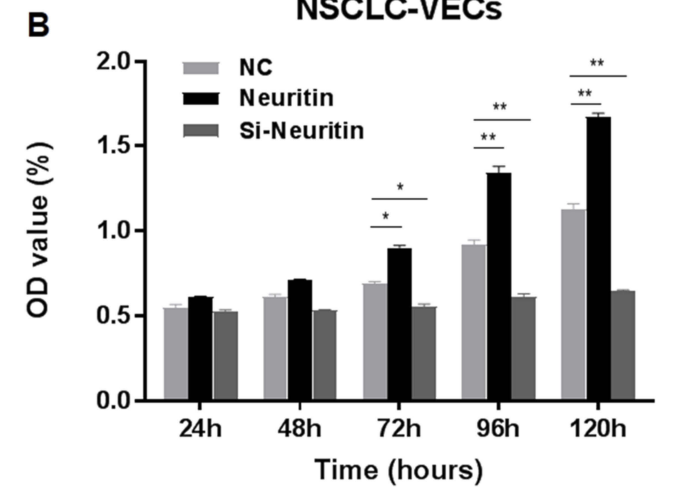

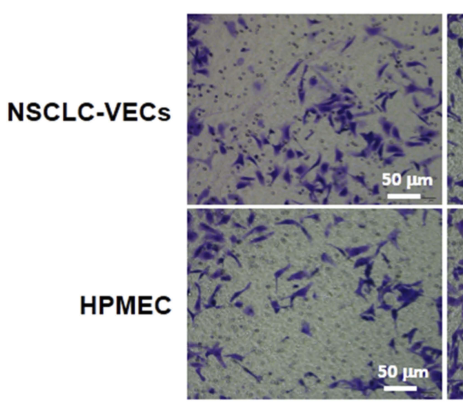

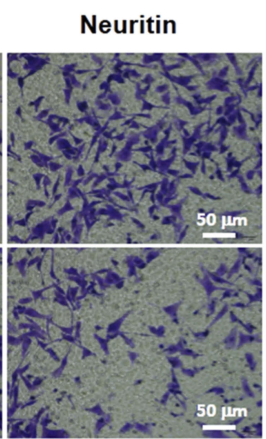

Si-Neuritin

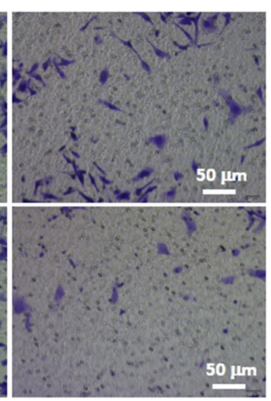

Figure 3 Neuritin promoted the proliferation and in vitro migration ability of HPMECs and NSCLC-VECs. Neuritin was overexpressed or silenced in HPMECs and NSCLCVECs. (A) Cell proliferation of HPMECs was detected with MTT. (B) Cell proliferation of NSCLC-VECs was detected. (C) Wound healing of HPMECs and NSCLC-VECs. (D) Changes in migration of HPMECs and NSCLC-VECs. Compared with NC group, ${ }^{*} p<0.05,{ }^{* *} p<0.01$.

(all $\mathrm{p}<0.05$ ) (Figure 4D and E). As a result, Neuritin overexpression promoted cell cycle progression and inhibited apoptosis of VECs.

\section{Neuritin Affects Cell Morphology}

Scanning electron microscopy analysis indicated that primary NSCLC-VECs and HPMECs appeared as short spindles. Cells were relatively large, highly refractive with fewer and shorter filopodia on the cell surface (Figure 5). Upon Neuritin overexpression, the increased number and thicker filopodia were observed on the surface of HPMECs and NSCLC-VECs, with few visible secondary bifurcations and enlarged terminal structures. These results suggested that Neuritin may improve vascular endothelial cell migration by promoting cell pseudo foot formation and enhancing intercellular adsorption.

\section{Discussion}

Neuritin is mainly expressed in human brain tissues, liver, and lungs whose regional distribution contributes to its participation in various biological processes. ${ }^{20}$ Neuritin expression is significantly elevated in VECs of tumor, ${ }^{21}$ such as Kaposi sarcoma and lung cancer. Still, the role and mechanism of abnormal Neuritin expression in tumor development and progression remain elusive. Therefore, our study attempts to examine the level of Neuritin expression in NSCLC and to determine the effect of dysregulated Neuritin expression on the biological behavior of VECs, exploring its possible potential mechanism of action.

Yuan et al found that the rate of strong Neuritin expression in gastric cancer tissues $(82.76 \%$ ) was notably upregulated in comparison with that in the adjacent normal tissues, which was consistent with the results found in lung cancer, colon cancer, prostate cancer, glioblastoma, and other solid tumors. ${ }^{16,22,23}$ According to the previous study results, it is reasonable to speculate that Neuritin expression might be associated with NSCLC-VECs. Consequently, the expression of Neuritin protein and mRNA in NSCLC-VECs and HPMECs was evaluated by Western blot analysis and qPCR. As expected, the Neuritin protein was highly expressed in NSCLC-VECs, suggesting that Neuritin may play a role in NSCLC development and 
A

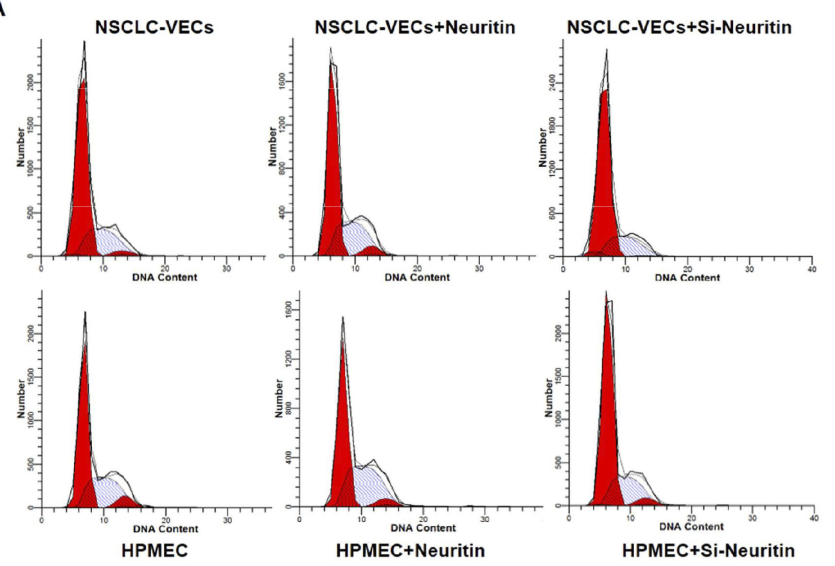

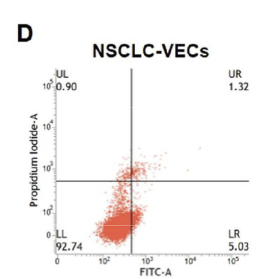
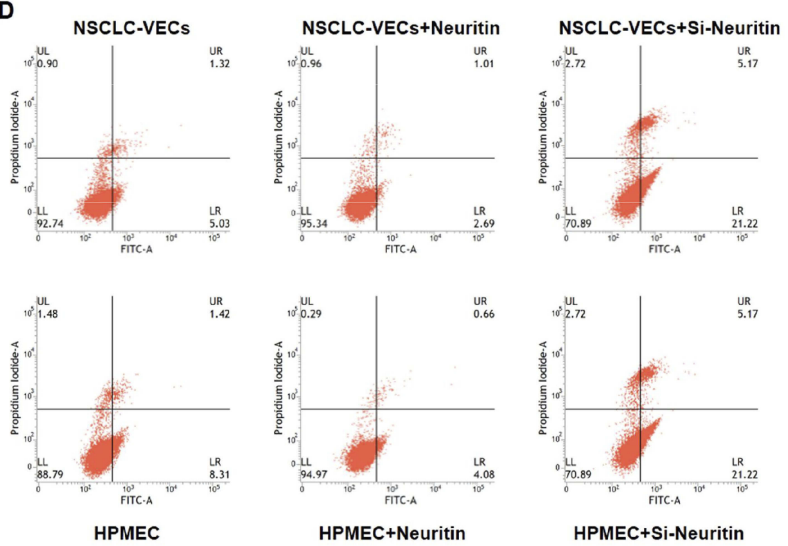

HPMEC+Si-Neuritin

E

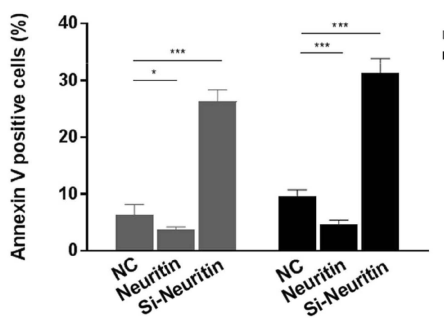

- NSCLC-VECs
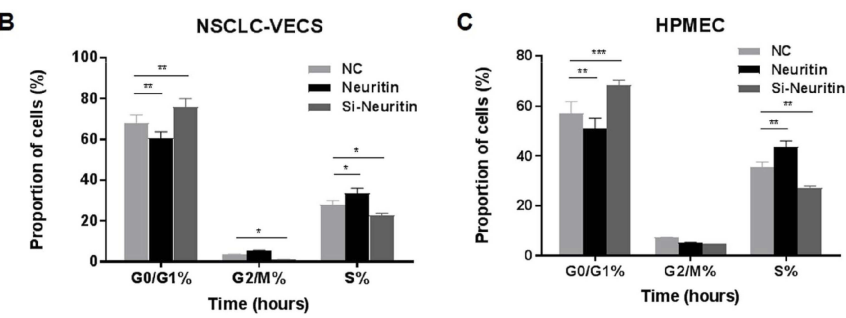

Figure 4 Neuritin regulated cell cycle and cell apoptosis in HPMECs and NSCLC-VECs. Neuritin was overexpressed or silenced in HPMECs and NSCLC-VECs. (A) Cell cycle distribution of HPMECs and NSCLC-VECs was analyzed by flow cytometry. (B and C) Cell cycle distribution was calculated. (D and E) Cell apoptosis was analyzed in HPMECs and NSCLC-VECs by flow cytometry. Compared with NC group, ${ }^{*} p<0.05,{ }^{*} *_{p}<0.01, *^{* * *} p<0.001$.

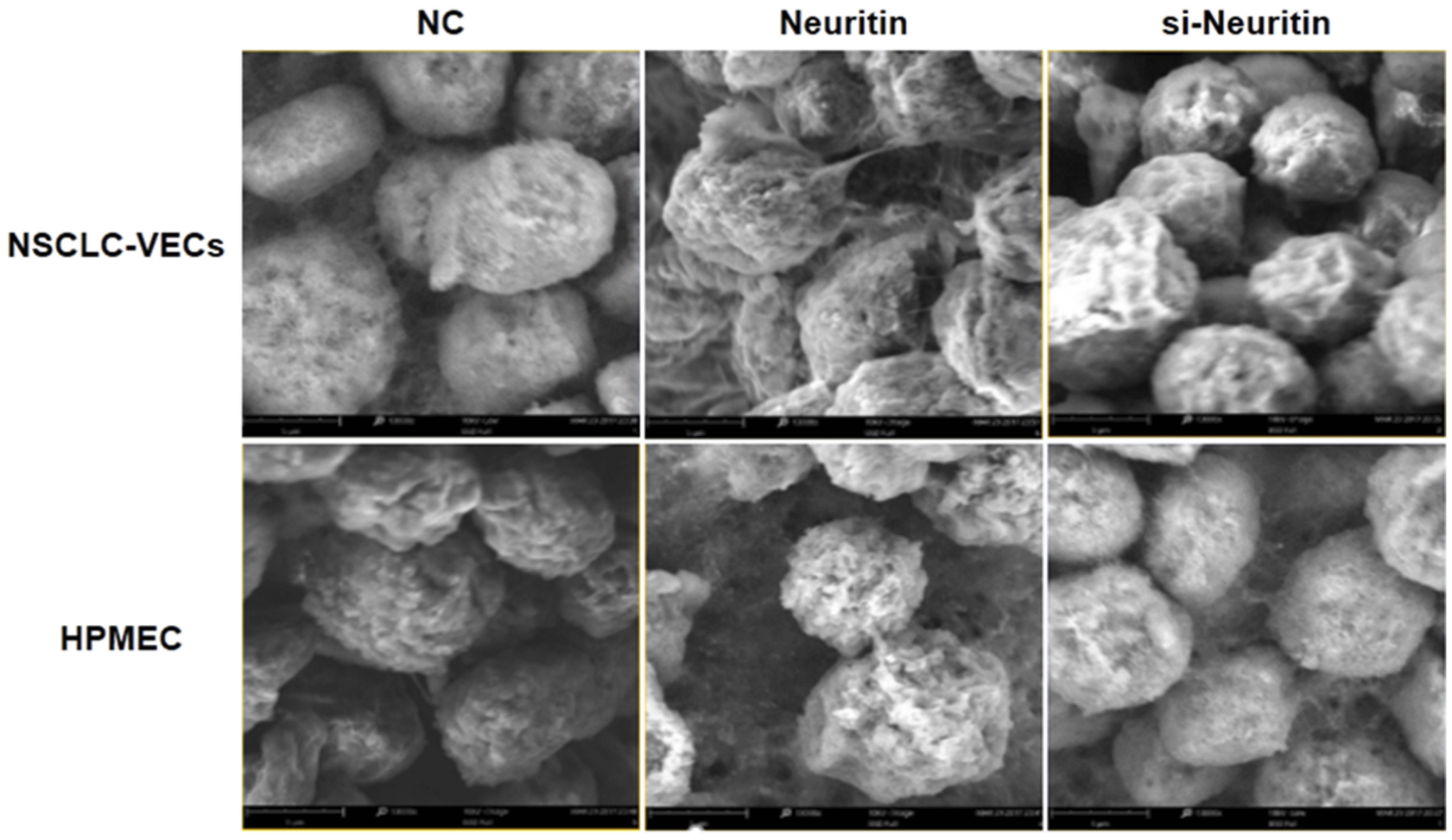

Figure 5 Changes in HPMEC and NSCLC-VEC morphology following Neuritin knockdown/overexpression as detected by scanning electron microscopy.

progression. It has been shown that Neuritin overexpression correlated positively with the malignancy of astrocytoma as reflected in promoting proliferation and inhibiting apoptosis. ${ }^{23}$ Consistent with this previous 
research, our study found that Neuritin significantly enhanced proliferative activity, wound healing and migration abilities of VECs, and reduced cell apoptosis. To the best of our knowledge, handful research exhibited the relation between the expression of Neuritin and the cell cycle. While we found that Neuritin overexpression promoted cell cycle progression of VECs, as indicated by increased transition from the G1 phase to the $S$ phase. Moreover, further confirmation by knocking down Neuritin suggested that Neuritin may be critical in tumorigenesis and progression of NSCLC by its biologic activities. However, the mechanism of action of Neuritin regulation in NSCLC-VEC is not clear.

VEGFR is an important regulator of tumor angiogenesis, activation of multiple intracellular signaling molecules can promote VEGFR signal transduction from the cell membrane, Tyr951 phosphorylation, and intracellular NO release, which facilitate VEC proliferation, migration, and angiogenesis of many tumors under hypoxic condition, resulting in the development, progression, invasion, and metastasis of malignant tumors. ${ }^{24,25}$ However, it is unclear whether the upregulation of VEGFR expression is synergistic with Neuritin. In addition, Neuritin protein is a negative regulator of Notch signaling pathway, and Notch signaling pathway and its associated receptor Notch1 are highly conserved cellular components. ${ }^{26}$ The Notch1 signaling pathway has also been shown to be closely related to the various biological processes of VECs in angiogenesis studies. ${ }^{27}$ Wang et $\mathrm{al}^{28}$ in earlier study demonstrated that knockdown of Notch-1 can effectively inhibit the growth and proliferation of colorectal cancer cells HT29, arrest the cell cycle at G1 phase, and promote apoptosis. Sha et al proved that curcumin-induced G0/G1 arrest and apoptosis in prostate cancer DU-145 cells by downregulating Notch signaling. ${ }^{29}$ Nevertheless, the activation of Notch-1 could lead to reactive astrogliosis after Intracerebral Hemorrhage. ${ }^{30}$ To better understand the role of Neuritin in VEGFR and Notch 1, VEGFR and Notch1 expression were assessed after Neuritin overexpression or knockdown in HPMECs and NSCLC-VECs. In the current study, overexpression of Neuritin upregulated VEGFR and downregulated Notch 1 expression. Hence, we considered that Neuritin might affect its biological activity by positively regulating VEGFR expression and negatively regulating Notch 1 signaling pathway. Finally, scanning electron microscopy examination demonstrated that Neuritin may also alter EC morphology to promote the biological function of cells during tumorigenesis.

\section{Conclusion}

In summary, differences of Neuritin expression detected in NSCLC-VECs and HPMEC cells suggest that Neuritin is associated with tumor angiogenesis. Neuritin may play a key role in the development and progression of NSCLC through its biological activities, including anti-apoptosis, promotion of VEC proliferation, migration, and cell cycle progression. In terms of potential molecular mechanisms, Neuritin may affect its biological activity by positively regulating VEGFR expression and negatively regulating Notch1 signaling. Neuritin may be a valuable biomarker for molecular diagnosis of NSCLC and can be severed as a potential target for the treatment of NSCLC. Further studies are needed in order to gain insight into the diagnostic and therapeutic efficiency of Neuritin in NSCLC.

\section{Acknowledgments}

This work was supported by Xinjiang Uygur Autonomous Region Municipal Natural Science Foundation (No. 2016D01C376) and Youth Medical Science and Technology Talents Special Scientific Research Project of the Health and Family Planning Commission in Xinjiang Uygur Autonomous Region (No. WJWY-201907).

\section{Disclosure}

The authors report no conflicts of interest in this work.

\section{References}

1. Katarzyna D, Krzysztof K, Satish G, et al. Serum folate concentration and the incidence of lung cancer. PLoS One. 2017;12:e177441. doi:10.1371/journal.pone.0177441

2. Hwan CJ. Immunotherapy for Non-small-cell lung cancer: current status and future obstacles. Immune Netw. 2017;17:378-391. doi:10. 4110/in.2017.17.6.378

3. Li C, Xue Y, Xi YR. Progress in the application and mechanism of metformin in treating non-small cell lung cancer. Oncol Lett. 2017;13:2873-2880. doi:10.3892/ol.2017.5862

4. Chou H-L, Lin Y-H, Liu W, et al. Combination therapy of chloroquine and $\mathrm{C} 2$-ceramide enhances cytotoxicity in lung cancer H460 and H1299 cells. Cancers. 2019;11:370. doi:10.3390/cancers 11030370

5. Wang J, Chen J, Guo Y, et al. Strategies targeting angiogenesis in advanced non-small cell lung cancer. Oncotarget. 2017;8:5385453872. doi:10.18632/oncotarget. 17957

6. Annese T, Tamma R, Ruggieri S, Ribatti D. Angiogenesis in pancreatic cancer: pre-clinical and clinical studies. Cancers. 2019;11:E381.

7. Wang Y, Liu J, Jiang Q, et al. Human adipose-derived mesenchymal stem cell-secreted CXCL1 and CXCL8 facilitate breast tumor growth by promoting angiogenesis. Stem Cells. 2017;35:2060. doi:10.1002/ stem.v35.9 
8. Mao G, Liu Y, Fang X, et al. Tumor-derived microRNA-494 promotes angiogenesis in non-small cell lung cancer. Angiogenesis. 2015;18:373-382. doi:10.1007/s10456-015-9474-5

9. Assareh E, Mehrnejad F, Mansouri K, et al. A cyclic peptide reproducing the $\alpha 1$ helix of VEGF-B binds to VEGFR-1 and VEGFR-2 and inhibits angiogenesis and tumor growth. BiochemJ. 2019. doi:10.1042/BCJ20180823

10. Cao W, Zhao F, Zhao Y, et al. Effect and correlation of VEGF and Notch1 in invasion and metastasis of breast cancer. J Basic Clin Oncol. 2012;8:2681-2692.

11. Moya-Horno I, Viteri S, Karachaliou N, et al. Combination of immunotherapy with targeted therapies in advanced non-small cell lung cancer (NSCLC). Ther Adv Med Oncol. 2018;10:175883401774501. doi:10.1177/1758834017745012

12. Serke M, Reinmuth N, Reck M. Targeted therapy in NSCLC against EGFR, ALK, OR VEGF. Atemwegs-Und Lungenkrankheiten. 2016;42:310-312. doi:10.5414/ATX02132

13. Wang $\mathrm{X}$, Liu $\mathrm{C}, \mathrm{Xu} \mathrm{F}$, et al. Effects of neuritin on the migration, senescence and proliferation of human bone marrow mesenchymal stem cells. Cell Mol Biol Lett. 2015;20:466-474. doi:10.1515/cmble2015-0026

14. Xu J, Cao W, Liu W, et al. Neuritin, a new potential target for optic nerve injury therapy. FASEB J. 2016;30:564.

15. Zhou S, Zhou J. Neuritin, a neurotrophic factor in nervous system physiology. Curr Med Chem. 2014;21. doi:10.2174/09298673216661 31218093327

16. Han D, Qin B, Liu G, et al. Characterization of neuritin as a novel angiogenic factor. Biochem Biophys Res Commun. 2011;415:608612. doi:10.1016/j.bbrc.2011.10.118

17. Le JS, Le MN, Cazes A, et al. Characterization of the expression of the hypoxia-induced genes neuritin, TXNIP and IGFBP3 in cancer. FEBS Lett. 2006;580:3395-3400. doi:10.1016/j.febslet.2006.05.011

18. Zhang P, Luo X, Guo Z, et al. Neuritin inhibits notch signaling through interacted with neuralized to promote the neurite growth. Front Mol Neurosci. 2017;10:179. doi:10.3389/fnmol.2017.00179

19. Zheng F, Wang S, Wen S, et al. Characterization and antibacterial activity of amoxicillin-loaded electrospun nano-hydroxyapatite/poly (lactic-co-glycolic acid) composite nanofibers. Biomaterials. 2013;34:1402-1412. doi:10.1016/j.biomaterials.2012.10.071
20. Cartledge JC, Rolland C, Lemerle S, et al. Novel cellular genes essential for transformation of endothelial cells by Kaposi's sarcoma-associated herpesvirus. Cancer Res. 2005;65:5084-5095. doi:10.1158/0008-5472.CAN-04-2822

21. Pan Z, Shi Z, Wei H, et al. Magnetofection based on superparamagnetic iron oxide nanoparticles weakens glioma stem cell proliferation and invasion by mediating high expression of MicroRNA-374a. $J$ Cancer. 2016;7:1487-1496. doi:10.7150/jca.15515

22. Yuan M, Li Y, Zhong C, et al. Overexpression of neuritin in gastric cancer. Oncol Lett. 2015;10:3832. doi:10.3892/ol.2015.3793

23. Zhang L, Zhao Y, Wang C-G, et al. Neuritin expression and its relation with proliferation, apoptosis, and angiogenesis in human astrocytoma. Med Oncol. 2011;28:907-912. doi:10.1007/s12032010-9537-9

24. Cascone T, Xu L, Lin HY, et al. The HGF/c-MET pathway is a driver and biomarker of VEGFR-inhibitor resistance and vascular remodeling in non-small cell lung cancer. Clin Cancer Res. 2017;23:54895501. doi:10.1158/1078-0432.CCR-16-3216

25. Huang X, Zhou G, Wu W, et al. Editing VEGFR2 blocks VEGFinduced activation of akt and tube formation. Invest Ophthalmol Vis Sci. 2017;58:1228-1236. doi:10.1167/iovs.16-20537

26. Shan T, Liu J, Wu W, et al. Roles of notch signaling in adipocyte progenitor cells and mature adipocytes. J Cell Physiol. 2017;232. doi:10.1002/jcp. 25697

27. Andrieu G, Tran AH, Strissel KJ, et al. BRD4 regulates breast cancer dissemination through Jagged1/Notch1 signaling. Cancer Res. 2016;76:6555. doi:10.1158/0008-5472.CAN-16-0559

28. Liao W, Li G, You Y, et al. Antitumor activity of Notch1 inhibition in human colorectal carcinoma cells. Oncol Rep. 2018;39:1063-1071. doi:10.3892/or.2017.6176

29. Sha J, Li J, Wang W, et al. Curcumin induces G0/G1 arrest and apoptosis in hormone independent prostate cancer DU-145 cells by down regulating Notch signaling. Biomed Pharmacother. 2016;84:177-184. doi:10.1016/j.biopha.2016.09.037

30. Zhong JH, Zhou HJ, Tang T, et al. Activation of the Notch-1 signaling pathway may be involved in intracerebral hemorrhage-induced reactive astrogliosis in rats. $J$ Neurosurg. 2017;129:1-8. doi:10.3171/ 2017.3.JNS162383
OncoTargets and Therapy

\section{Publish your work in this journal}

OncoTargets and Therapy is an international, peer-reviewed, open access journal focusing on the pathological basis of all cancers, potential targets for therapy and treatment protocols employed to improve the management of cancer patients. The journal also focuses on the impact of management programs and new therapeutic agents and protocols on patient perspectives such as quality of life, adherence and satisfaction. The manuscript management system is completely online and includes a very quick and fair peer-review system, which is all easy to use. Visit http://www.dovepress.com/ testimonials.php to read real quotes from published authors. 